\title{
Antenna Beamwidths of Above-The-Road Radar for Traffic Speed Enforcement in China
}

\author{
Lei Du, Qiao Sun, Changqing Cai, Yue Zhang, and Hongbo Hu \\ Division of Mechanics and Acoustics, National Institute of Metrology, Beijing 100013, China \\ Correspondence should be addressed to Lei Du, dulei@nim.ac.cn
}

Received 26 March 2012; Revised 2 June 2012; Accepted 12 June 2012

Academic Editor: Charles Bunting

Copyright () 2012 Lei Du et al. This is an open access article distributed under the Creative Commons Attribution License, which permits unrestricted use, distribution, and reproduction in any medium, provided the original work is properly cited.

\begin{abstract}
Above-The-Road (AbTR) radar is a new type of speed measurement device used in China for traffic speed enforcement. The antenna beamwidths in vertical and horizontal directions are critical parameters for AbTR radar because speed measurement accuracy partly depends on vertical beamwidth and speed measurement interference from adjacent lanes can be effectively minimized by appropriate choice of horizontal beamwidth. In this paper, the speed measurement principle of AbTR radar is introduced and the reason for field speed measurement error is presented. The speed measurement error is theoretically calculated in various combinations of different vertical beamwidths and possible installation angles. The requirement for horizontal beamwidth to operate with less interference from adjacent lanes is also theoretically laid down with different installation heights and angles. Field tests with three equal-distanced piezosensors each lane as triggering references have been performed on an AbTR radar sample with vertical beamwidth $5.1^{\circ}$ and horizontal beamwidth $5.2^{\circ}$. The results of theoretical analysis and field tests validate that the requirement of less than $6^{\circ}$ for AbTR radar antenna beamwidths in vertical and horizontal directions is a precondition for accurate and reliable field measurements, which has been added to the China's pattern approval regulation for AbTR radar devices newly published.
\end{abstract}

\section{Introduction}

Above-The-Road (AbTR) radar is a new type of vehicle speed measurement device used in China for traffic speed law enforcement. Compared with the traditional AcrossThe-Road (AcTR) radar $[1,2]$ and Down-The-Road (DTR) radar $[3,4]$, which are the common types of traffic speed measurement devices widely used in China and other places of the world $[2,4]$, the advantage of AbTR radar is that it is fixed at a known angle above the road along a specified single lane with less interference from adjacent lanes and functions with a variable speed limit sign in the heavy traffic and multilane highway.

As the number of vehicles increases rapidly in China, AcTR radar and DTR radar cannot fully meet the law enforcement demands of traffic police. AbTR radar has been used to measure the speed of vehicles and record the offenders quickly and automatically by working together with a camera in certain provinces of China. However, abnormal field measurement results occasionally occurred with some AbTR radar devices, which had been approved according to the former related pattern approval [5] regulation in China. These abnormal results would have resulted in improper fine on drivers if they had passed manual check. One reason for this was that antenna metrological parameters of AbTR radar were not required in the former related pattern approval regulation. Additionally, the former field test method could not be used to help operators to effectively distinguish the unqualified AbTR radar devices. Therefore, a special pattern approval regulation for AbTR radar has been officially approved in China to ensure the measurement results of AbTR radar devices more accuratly and reliably. The major modifications in the new pattern approval regulation include the requirement for antenna beamwidths and the metrological field test in actual traffic, which is recommended in OIML R 91 [5].

In this paper, we are concerned with the influence of antenna beamwidths of AbTR radar on traffic speed 
measurement results, including vertical beamwidth on speed measurement error and horizontal beamwidth on interference from adjacent lanes. The paper is organized as follows. The speed measurement principle of AbTR radar is introduced in Section 2. The reason for the field speed measurement error of AbTR radar is analyzed in Section 3. The AbTR radar antenna beamwidths, which will be mandatory requirements for AbTR radar used in China for traffic speed law enforcement, are proposed by theoretical calculation in various combinations of installation parameters in Section 4. A field test in actual traffic is designed and performed on an AbTR radar sample, whose antenna beamwidths meet the requirements given in Section 4, to validate the theoretical analysis results in Section 5. The conclusions are made in Section 6.

\section{Principle of Speed Measurement}

As shown in Figure 1, AbTR radar is typically installed on the gantry above the road, and the radar antenna is directed to the specified single lane at a known installation angle $\theta$, which is the angle between the horizontal plane parallel to the road surface on which the target vehicle is travelling and the beam direction of the radar antenna. Reference line in Figure 1 is corresponding to the ground position of the installation angle $\theta$. The main difference of AbTR radar from AcTR radar and DTR radar is that the AbTR radar shall work only when the target vehicle passes the measurement region at its specified lane and not detect any vehicles travelling in the adjacent lanes.

AbTR radar uses the Doppler effect to measure the speed of target vehicle. AbTR radar transmits an unmodulated continuous microwave beam at frequency $f_{0}$. When a moving vehicle travels through the microwave beam, AbTR radar receives the microwave signal reflected from the target vehicle at frequency $f_{1}$ and processes the received signal by mixing with the transmitted signal. The frequency difference between the transmitted frequency $f_{0}$ and the received frequency $f_{1}$ is the Doppler shift $f_{d}$, which is directly proportional to the target vehicle speed $v$ and can be written as

$$
f_{d}=\frac{2}{C} \cdot f_{0} \cdot v \cdot \cos \varphi,
$$

where $C$ is the speed of electromagnetic wave propagating in air, $v \cdot \cos \varphi$ denotes the radial velocity of the target vehicle relative to AbTR radar, and $\varphi$ is defined as the triggering angle, which is the included angle between the direction of target vehicle movement and the direction from the AbTR radar to the target vehicle at the triggering point, as shown in Figure 1. Triggering line in Figure 1 is corresponding to the ground position of the triggering angle $\varphi$.

Doppler shift $f_{d}$ is positive when the target vehicle approaches AbTR radar, and negative when the target vehicle recedes from AbTR radar. The value of Doppler shift $f_{d}$ is in the audio frequency range from several hundred $\mathrm{Hz}$ to tens of thousands $\mathrm{Hz}$ and can be computed by the digital signal processing (DSP) unit of AbTR radar. The value of transmitted frequency $f_{0}$ is also known, which is dependent

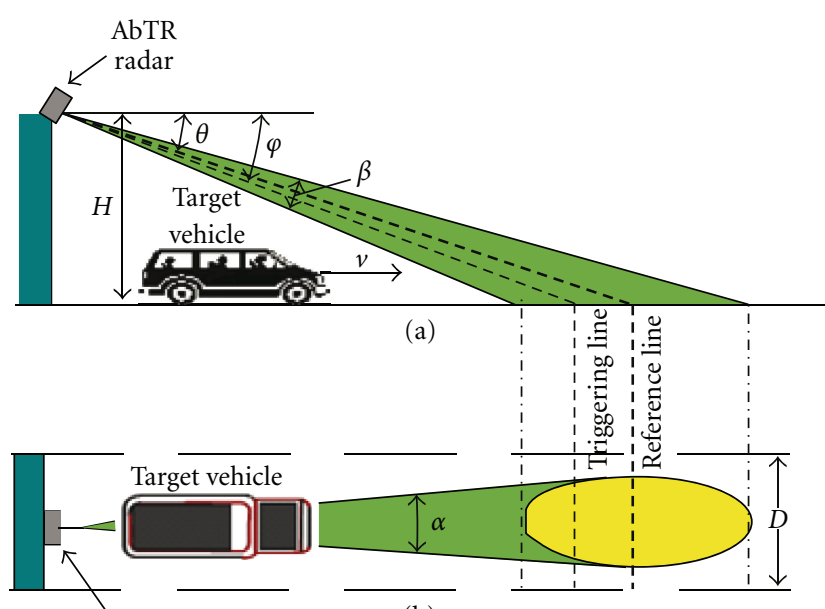

(b)

FIGURE 1: Diagram of AbTR radar speed measurement principle. (a) Side view and (b) Top view.

on the local oscillator unit of AbTR radar. If the value of triggering angle $\varphi$ can also be known by measurement or computation, then the theoretical value of target vehicle speed $v$ is given by

$$
v=\frac{C}{2 \cos \varphi} \cdot \frac{f_{d}}{f_{0}} .
$$

\section{Analysis of Speed Measurement Error}

However, the value of triggering angle $\varphi$ is unknown and not the same for different target vehicles. It depends on many factors, typically including types of the target vehicle and technical parameters of AbTR radar.

One of the most important factors is the radar crosssection (RCS) [6] of the target vehicle relative to AbTR radar. Since most AbTR radars use the same antenna for transmitting and receiving, the backscatter RCS of target vehicle is the usual case of interest for AbTR radar. Backscatter RCS is a complicated multiparameter function, including position of AbTR radar relative to target vehicle, angular orientation of target vehicle relative to AbTR radar, installation angle of AbTR radar, microwave frequency band of AbTR radar, polarization of transmitter and receiver, geometry and material composition of target vehicle, and so forth. Firstly, the installation parameters of different AbTR radars, such as height, distance, and angle, are decided by each manufacturer and not the same in the usual case. Secondly, AbTR radar usually operates within allocated narrow frequency range in one of three broad frequency bands: $\mathrm{X}$ band (nominally $8 \mathrm{GHz}$ to $12 \mathrm{GHz}$ in China), $\mathrm{K}$ band (nominally $18 \mathrm{GHz}$ to $26.5 \mathrm{GHz}$ in China), and $\mathrm{Ka}$ band (nominally $26.5 \mathrm{GHz}$ to $40 \mathrm{GHz}$ in China). Propagation effect and target reflectivity are similar for frequencies within the same band, but may be very different from one band to another. Thirdly, polarization is one of the fundamental characteristics of any antenna, and the main types of antenna polarization include linear polarization, circular 
polarization, and elliptical polarization. The backscatter RCS of the same target vehicle is also different when AbTR radars use the one of the three different polarization types. Finally, there are many different types of target vehicle in actual traffic, such as truck, car, motorcycle, and bus. Various types of target vehicle differentiate each other in size, shape, and material, which result in the different backscatter RCS values relative to the same AbTR radar.

For the above reasons, it is impossible for present AbTR radar technology to measure the backscatter RCS in each case precisely, which means that the precise value of triggering angle $\varphi$ cannot be known either. According to (2), the unknown triggering angle $\varphi$ leads to the fact that AbTR radar cannot measure the actual value of target vehicle speed, and only some approximation can be found to obtain the measurement value of target vehicle speed. One of the most widely used approximation method at present is to use the known installation angle $\theta$, instead of the unknown triggering angle $\varphi$, for all the speed measurements of target vehicles. Based on this approximation, we get the approximate value of target vehicle speed measurement, $v_{m}$, which can be written as

$$
v_{m}=\frac{C}{2 \cos \theta} \cdot \frac{f_{d}}{f_{0}} .
$$

According to (2) and (3), the relation between the actual value of target vehicle speed and the measured value given by AbTR radar is

$$
v_{m}=v \cdot \frac{\cos \varphi}{\cos \theta} .
$$

The relative measurement error of target vehicle speed, $\delta_{v}$, is given by

$$
\delta_{v}=\frac{v_{m}-v}{v}=\frac{\cos \varphi}{\cos \theta}-1 .
$$

From (5), we can conclude that the relative measurement error $\delta_{v}$ is theoretically decided by the difference between the triggering angle $\varphi$ and the installation angle $\theta$. The closer the triggering angle $\varphi$ is to the installation angle $\theta$, the smaller the relative measurement error will be.

Another important influence factor of the triggering angle $\varphi$ is the technical performance of AbTR radar antenna, which can be described by antenna radiation pattern as shown in Figure 2. Beamwidth and sidelobe level are the two key metrological parameters of radar antenna for the pattern approval of radar for traffic speed enforcement $[1,3,5]$.

Antenna beamwidth describes the width of mainlobe in the antenna radiation pattern. A common definition is the half-power beamwidth or the $-3 \mathrm{~dB}$ beamwidth, which is defined as the angular distance between the half-power points on each side of the peak in the mainlobe of antenna radiation pattern as shown in Figure 2. Beamwidth is the major influence factor to the speed measurement accuracy and will be discussed in detail in the latter part of this paper.

Sidelobes are the other lobes except the mainlobe in the antenna radiation pattern as shown in Figure 2 and usually represent unwanted radiation in undesired directions. Sidelobe level is the peak of the sidelobes relative to the peak

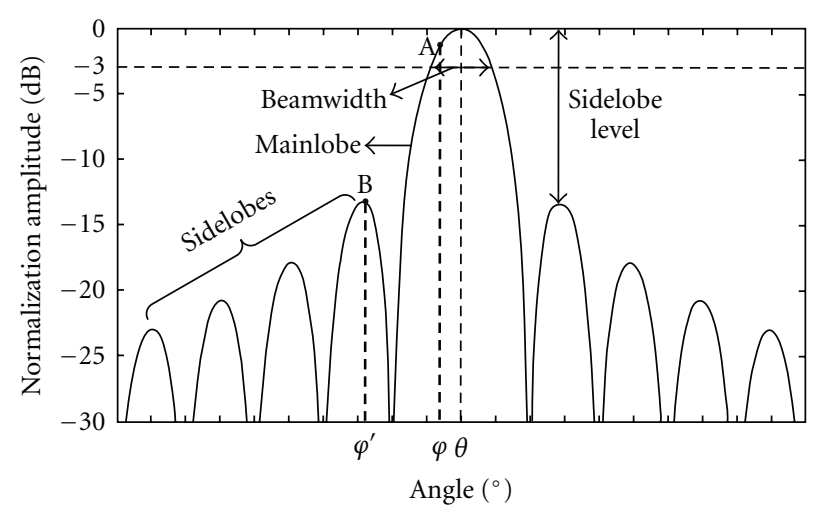

Figure 2: Antenna radiation pattern.

of the mainlobe. Generally, it is desirable to minimize the sidelobe level of radar for traffic speed enforcement to avoid the erroneous triggering in the undesired angle $\varphi^{\prime}$ (marked $B$ in Figure 2). After the theoretical analysis and a large quantity of field tests in actual traffic, it has been proven by the metrologists that the sidelobe level of radar for traffic speed enforcement must be less than $-15 \mathrm{~dB}$ in any direction to avoid possible erroneous triggering $[1,3,5]$. As a new type of radar for traffic speed enforcement, the above conclusion is also tenable for AbTR radar.

\section{Antenna Beamwidths}

Although the speed measurement error is unavoidable for present AbTR radar technology due to the unknown triggering angle $\varphi$ of each target vehicle as analyzed in Section 3, the speed measurement accuracy can be improved by reducing the error between the installation angle $\theta$ and the triggering angle $\varphi$, which is related to the antenna vertical beamwidth of AbTR radar. Additionally, the measuring region of AbTR radar is restricted to the specified single lane, which is dependent on the antenna horizontal beamwidth of AbTR radar. In this section, the influences of antenna beamwidths of AbTR radar on traffic speed measurement results, including vertical beamwidth on speed measurement error and horizontal beamwidth on interference from adjacent lanes, are analyzed, respectively, and the values of antenna beamwidths are proposed by theoretical calculation in various combinations of installation parameters.

4.1. Vertical Beamwidth. Vertical beamwidth is a key metrological technical parameter of AbTR radar, which denotes the antenna beamwidth in the along-the-road direction and represented by $\beta$ as shown in Figure 1(a). The relation between vertical length of speed measurement region and vertical beamwidth of antenna radiation pattern is shown in Figure 3. The radiated power reaches its maximum in the direction of installation angle $\theta$ and becomes lower with the angle farther from $\theta$. Since the target vehicle with a high backscatter RCS (e.g., a heavy truck with bare metal material, rough surface, large size, and square shape) reflects more electromagnetic energy back to the AbTR radar than the 


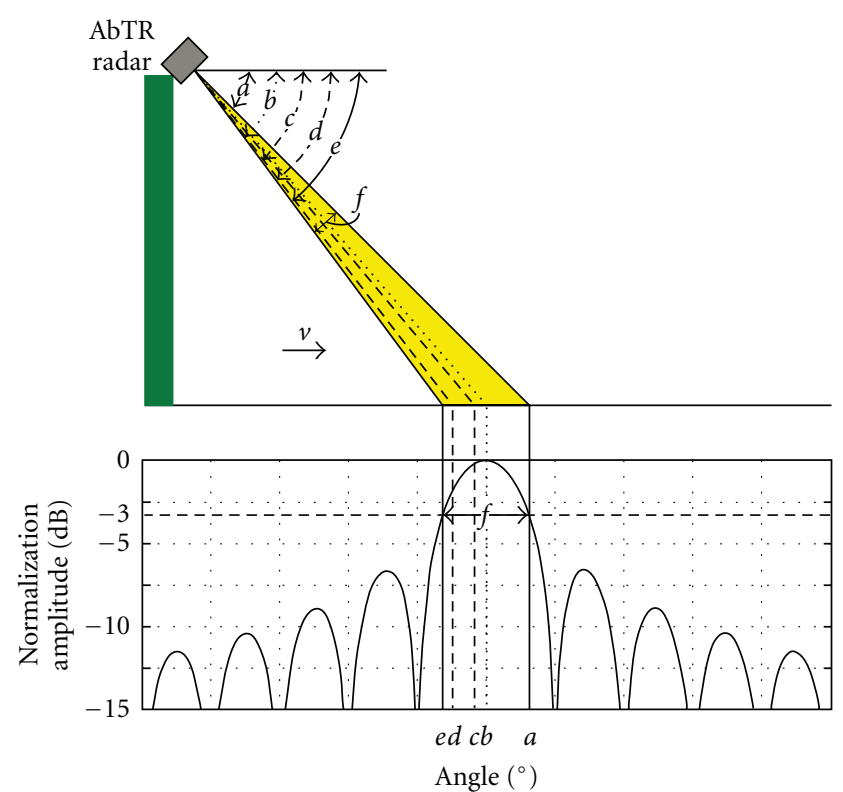

Figure 3: Relation between vertical length of speed measurement region and vertical beamwidth of antenna radiation pattern, where $a=\theta-\beta / 2, b=\theta, c=\varphi_{1}, d=\varphi_{2}, e=\theta+\beta / 2$, and $f=\beta$.

one with a low backscatter RCS (e.g., a car with synthetic material, smooth surface, small size, and streamlined shape), the former can be detected by the AbTR radar at the angle far from the installation angle $\theta$, which is represented by $\varphi_{2}$ in Figure 3, while the latter must be detected at the angle close to the installation angle $\theta$, which is represented by $\varphi_{1}$ in Figure 3. It has been analyzed in Section 3 that present AbTR radar cannot distinguish $\varphi_{1}$ from $\varphi_{2}$, but use the same installation angle $\theta$ instead in actual traffic speed measurement, which is the major influence factor to the speed measurement accuracy of field operation.

In order to restrict the maximum speed measurement error and assure the traffic court's confidence in using AbTR radar devices for speeding punishment, a mandatory requirement for the certificated AbTR radar in China is that it should acquire data only when the target vehicle passes through the antenna vertical beamwidth, which means that the range of triggering angle $\varphi$ should be within the range shown below:

$$
\theta-\frac{\beta}{2} \leq \varphi \leq \theta+\frac{\beta}{2} .
$$

Equation (5) and inequation (6) show that the maximum speed measurement error is mainly related to the antenna vertical beamwidth of AbTR radar. The wider the antenna vertical beamwidth is, the larger the maximum speed measurement error will be. Apart from that, the installation angle also has some influence on the maximum speed measurement error. The relative measurement errors of target vehicle speed are theoretically calculated with different antenna vertical beamwidths and possible installation angles of AbTR radar widely used in China, where the antenna vertical beamwidth $\beta$ is chosen as $4^{\circ}, 6^{\circ}, 8^{\circ}, 10^{\circ}, 12^{\circ}, 14^{\circ}, 16^{\circ}$, and $18^{\circ}$, respectively, and the installation angle $\theta$ is chosen as $20^{\circ}, 22.5^{\circ}, 25^{\circ}$, and $30^{\circ}$, respectively. Based on (5), the calculation values of relative errors are given in Table 1 .

The theoretical calculation results in Table 1 show that the antenna vertical beamwidth $\beta$ of the certificated AbTR radar should not exceed $6^{\circ}$ to meet the requirements for speed accuracy of field operation in China, that is, the relative error of target vehicle speed measurement by AbTR radar should be within $\pm 3 \%$. The mandatory requirement that antenna vertical beamwidth of AbTR radar must be smaller than $6^{\circ}$ has been added to the pattern approval regulation of China newly published and will also be added to the national AbTR radar standard of China in the future.

4.2. Horizontal Beamwidth. Horizontal beamwidth is another key metrological technical parameter of AbTR radar, which denotes the antenna beamwidth in the across-the-road direction and represented by $\alpha$ as shown in Figure 1(b). The relation between horizontal width of speed measurement region and horizontal beamwidth of antenna radiation pattern is shown in Figure 4. For the AbTR radar with broadbeam antenna shown in Figure 4(a), since its measuring region exceeds the specified single lane and covers the adjacent two lanes, the vehicle passing the measurement point in adjacent lanes may be detected by mistake. For example, the AbTR radar in Figure 4(a) detects a car travelling in the adjacent lane (marked A in Figure 4(a)) by mistake and measures the speed of the car while the identification of offender provided by the camera used for photographic evidence is a truck (marked B in Figure 4(a)). If the car exceeds the speed limit, the result is the wrong fine on the truck.

To avoid the wrong fine mentioned above, AbTR radar shall use a narrow-beam antenna (shown in Figure 4(b)) to only detect the vehicles travelling in the specified lane and avoid detecting any vehicles passing the measurement point in adjacent lanes. To ensure that the measuring region of AbTR radar is restricted to the specified single lane, a requirement for the certificated AbTR radar in China is that the covering width of antenna horizontal beamwidth shall be not more than two-thirds of the lane width at a given distance [7], which means that the following inequation should be satisfied according to the geometrical relationships in Figure 1:

$$
2 \cdot \frac{H}{\sin \theta} \cdot \tan \frac{\alpha}{2} \leq \frac{2}{3} D
$$

where $H$ is the installation height, and $D$ is the lane width. According to inequation (7), antenna horizontal beamwidth $\alpha$ of the certificated AbTR radar should satisfy

$$
\alpha \leq 2 \cdot \arctan \left(\frac{\sin \theta}{3} \cdot \frac{D}{H}\right) .
$$

The maximum permissible antenna horizontal beamwidth of the certificated AbTR radar devices is theoretically calculated according to inequation (8) with different installation heights and angles of AbTR radar widely used in China, where the installation height $H$ is chosen at $6 \mathrm{~m}$, 
TABLE 1: Theoretical calculation of relative error in various combinations of installation angle and vertical beamwidth.

\begin{tabular}{|c|c|c|c|c|c|c|c|c|}
\hline \multirow{2}{*}{$\theta$} & \multicolumn{8}{|c|}{$\beta$} \\
\hline & $4^{\circ}$ & $6^{\circ}$ & $8^{\circ}$ & $10^{\circ}$ & $12^{\circ}$ & $14^{\circ}$ & $16^{\circ}$ & $18^{\circ}$ \\
\hline $20^{\circ}$ & $-1.3 \% \sim 1.2 \%$ & $-2.0 \% \sim 1.8 \%$ & $-2.8 \% \sim 2.3 \%$ & $-3.6 \% \sim 2.8 \%$ & $-4.4 \% \sim 3.3 \%$ & $-5.2 \% \sim 3.7 \%$ & $-6.0 \% \sim 4.1 \%$ & $-6.9 \% \sim 4.5 \%$ \\
\hline $22.5^{\circ}$ & $-1.5 \% \sim 1.4 \%$ & $-2.3 \% \sim 2.0 \%$ & $-3.1 \% \sim 2.6 \%$ & $-4.0 \% \sim 3.2 \%$ & $-4.9 \% \sim 3.8 \%$ & $-5.8 \% \sim 4.3 \%$ & $-6.7 \% \sim 4.8 \%$ & $-7.7 \% \sim 5.2 \%$ \\
\hline $25^{\circ}$ & $-1.7 \% \sim 1.6 \%$ & $-2.6 \% \sim 2.3 \%$ & $-3.5 \% \sim 3.0 \%$ & $-4.4 \% \sim 3.7 \%$ & $-5.4 \% \sim 4.3 \%$ & $-6.4 \% \sim 4.9 \%$ & $-7.5 \% \sim 5.5 \%$ & $-8.5 \% \sim 6.1 \%$ \\
\hline $30^{\circ}$ & $-2.1 \% \sim 2.0 \%$ & $-3.0 \% \sim 2.9 \%$ & $-4.3 \% \sim 3.8 \%$ & $-5.4 \% \sim 4.7 \%$ & $-6.6 \% \sim 5.5 \%$ & $-7.8 \% \sim 6.3 \%$ & $-9.0 \% \sim 7.1 \%$ & $-10.3 \% \sim 7.8 \%$ \\
\hline
\end{tabular}

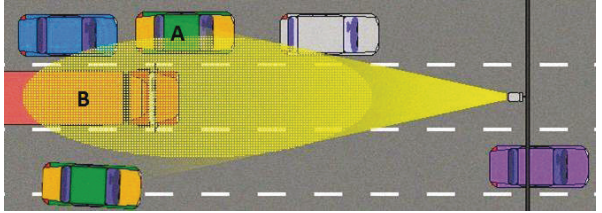

(a)

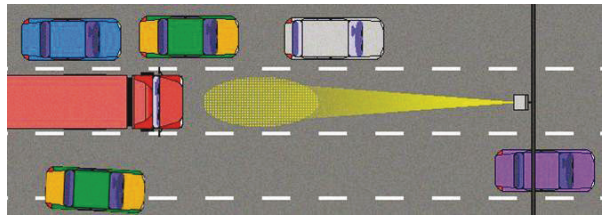

(b)

FIGURE 4: Relation between horizontal width of speed measurement region and horizontal beamwidth of antenna radiation pattern. (a) AbTR radar with broad beam antenna and (b) AbTR radar with narrow beam antenna.

TABLE 2: Theoretical calculation of maximum permissible antenna horizontal beamwidth of AbTR radar devices.

\begin{tabular}{lcccc}
\hline$H$ & & & $\theta$ & \\
& $20^{\circ}$ & $22.5^{\circ}$ & $25^{\circ}$ & $30^{\circ}$ \\
\hline $6 \mathrm{~m}$ & $8.2^{\circ}$ & $9.1^{\circ}$ & $10.1^{\circ}$ & $11.9^{\circ}$ \\
$7 \mathrm{~m}$ & $7.0^{\circ}$ & $7.8^{\circ}$ & $8.6^{\circ}$ & $10.2^{\circ}$ \\
$8 \mathrm{~m}$ & $6.1^{\circ}$ & $6.8^{\circ}$ & $7.6^{\circ}$ & $8.9^{\circ}$ \\
\hline
\end{tabular}

$7 \mathrm{~m}$, and $8 \mathrm{~m}$, respectively, the installation angle $\theta$ is chosen at $20^{\circ}, 22.5^{\circ}, 25^{\circ}$, and $30^{\circ}$, respectively, and the lane width $D$ equals to $3.75 \mathrm{~m}$ according to the national highway standard of China. The calculation results of maximum permissible horizontal beamwidth are given in Table 2.

The theoretical calculation results in Table 2 show that the antenna horizontal beamwidth $\alpha$ of the certificated AbTR radar should not exceed $6^{\circ}$ to meet the different installation requirements in China. The recommended requirement that antenna horizontal beamwidth of AbTR radar should be less than $6^{\circ}$ will be added to the national AbTR radar standard of China in the future.

\section{Experimental Validation}

It is recommended in OIML R 91 that the metrological field tests should be completed by an operating test in actual traffic with the advantage of the overall study of possible errors due to the complexity of factors affecting the result of measurement, such as shape of antenna pattern, lateral distance between AbTR radar and target vehicle, reflection characteristics of target vehicle, change of lane during target vehicle passage through radar beam, braking or accelerating, and presence of more than one vehicle [5]. Therefore, a field test has been designed and performed by an operating test in actual traffic to validate the previous theoretical analysis about antenna beamwidth of AbTR radar, in which the antenna beamwidths of the chosen AbTR radar sample in the vertical and horizontal plane are both designed to be less than $6^{\circ}$.

\subsection{Traffic Speed Measurement Standard System of Field} Operation. To meet the recommendation in OIML R 91 about the metrological field tests in actual traffic, a traffic speed measurement standard system of field operation (abbreviated to standard system thereafter) has newly been set up at number S16 Expressway from Hangzhou to Shanghai in China, which is a trilane freeway with speed limit $120 \mathrm{~km} / \mathrm{h}$. The schematic layout of the standard system is shown in Figure 5(a), and the photograph is shown in Figures 5(b) and 5(c). The standard system is composed of six parts. The first part includes five groups of three equaldistanced piezosensors, which can provide the reference triggering signals of target vehicle traveling in different lane and are represented by $1-5$ in Figure 5 . The second part includes three groups of two inductive loop sensors, which are preburied for the field test of inductive loop speed meter samples and represented by 6-8 in Figure 5 . The third part is a gantry with the height $8 \mathrm{~m}$ represented by 9 in Figure 5 , which is used for the installation of AbTR radar samples. The fourth part is a cabinet represented by 10 in Figure 5, which is used for the placement of signal processors of piezo speed meters to obtain the speed measurements, and for power supply of the whole standard system. The fifth part is a ground platform represented by 11 in Figure 5, which is used for the placement of AcTR radar samples. The sixth part is the platform used for the placement of the AbTR radar sample in this field test and represented by 12 in Figure 5.

The five piezo speed meters in the standard system are the same and can provide the reference speed values of target vehicles at five different places in three lanes, in which piezo speed meters 1,2 , and 3 can provide the reference speed values of target vehicles travelling in three different lanes simultaneously for the AbTR radar samples installed at the gantry 9, while piezo speed meters 1,4 , and 5 can provide the reference speed values of target vehicles 


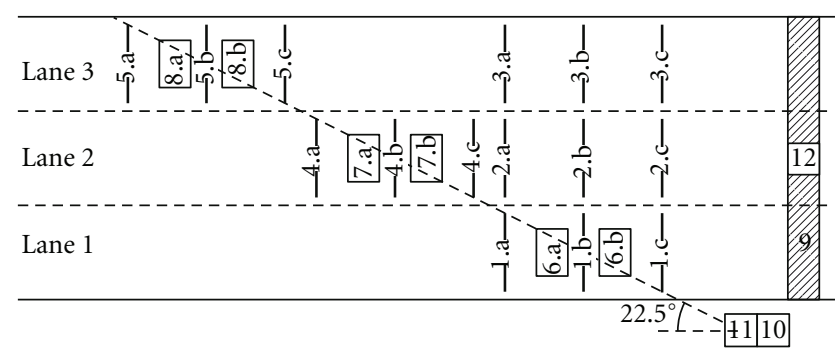

(a)

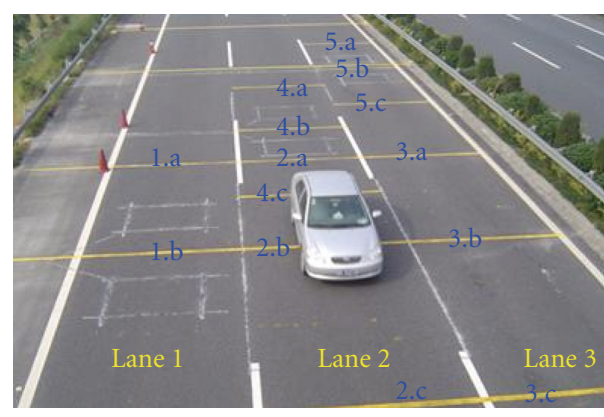

(b)

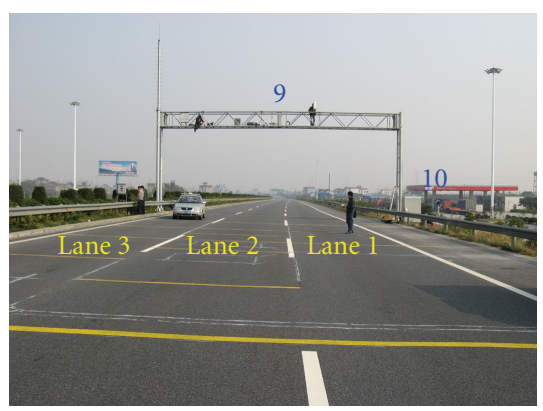

(c)

FIGURE 5: Traffic speed measurement standard system of field operation. (a) Schematic layout; (b) photograph from the gantry; (c) photograph from the ground.

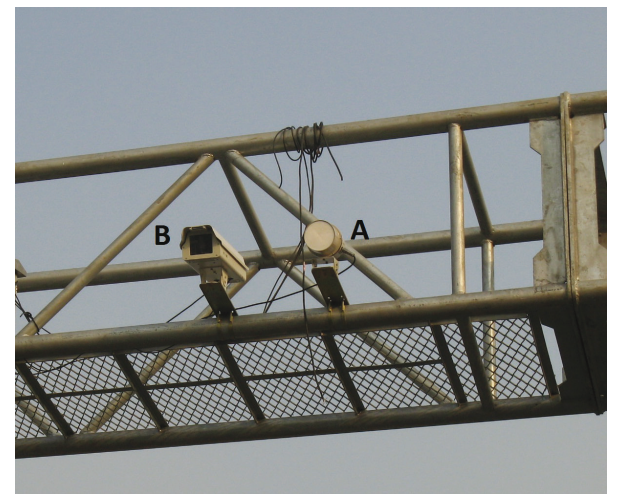

Figure 6: Photograph of the chosen AbTR radar sample.

travelling in three different lanes simultaneously for the AcTR radar samples placed at the ground platform 11 in the metrological field tests. All the five piezo speed meters have three piezosensors, a, b and c, with equal distance $6 \mathrm{~m}$ as shown in Figure 5. The region between piezosensor a and $\mathrm{c}$ is taken as the metrological test region. It should be noted that the measurement region of speed meter sample must be adjusted to the same within the metrological test region.

When a target vehicle travels through the test region, the piezosensor $\mathrm{a}, \mathrm{b}$ and $\mathrm{c}$ are pressed by the wheels of target vehicle sequentially, and the triggering signals generated by the three piezosensors are recorded and processed by the signal processors of piezo speed meter placed at the cabinet 10. By calculating the time intervals between piezosensors $\mathrm{a}$, and $\mathrm{b}$, the average speed value of target vehicle travelling through a and $\mathrm{b}, v_{\mathrm{ab}}$, can be measured by the piezo speed meter. Likewise, the average speed value of target vehicle travelling through $\mathrm{b}$ and $\mathrm{c}, \nu_{\mathrm{bc}}$, can also be measured by calculating the time intervals between piezosensors $\mathrm{b}$ and $\mathrm{c}$. The two measurement results $\nu_{\mathrm{ab}}$ and $\nu_{\mathrm{bc}}$ are independent and uncorrelated. When the difference between $v_{\mathrm{ab}}$ and $\nu_{\mathrm{bc}}$ lies in the maximum permissible error set manually, this test result is valid and the average speed value of target vehicle travelling through a and c, $v_{\mathrm{ac}}$, is taken as the reference speed value. The speed measurement error of the speed meter sample can be calculated by comparison with the reference speed value $v_{\mathrm{ac}}$.

The reference speed value provided by the standard system can be traced to the national primary standard of length and time in China. The relative expanded uncertainty of reference speed measurement is $U_{\text {rel }}=0.69 \%(k=3)[8-$ $10]$, which can meet the requirement for AbTR radar speed measurement accuracy in China, that is, the relative error of speed measurement should be within $\pm 3 \%$.

5.2. Field Test Results of the AbTR Radar Sample. To include the possible effects of vehicles travelling in the left or right of the test lane on the performance of the AbTR radar, the region between piezosensors $4 . \mathrm{a}$ and $4 . \mathrm{c}$ at the middle lane (see Figure 5) is chosen as the metrological test region of the AbTR radar sample. The photograph of the AbTR radar sample in this field test is shown in Figure 6, where A is the AbTR radar and B is the camera for photographic evidence. The sample is specially designed for this field test, whose 


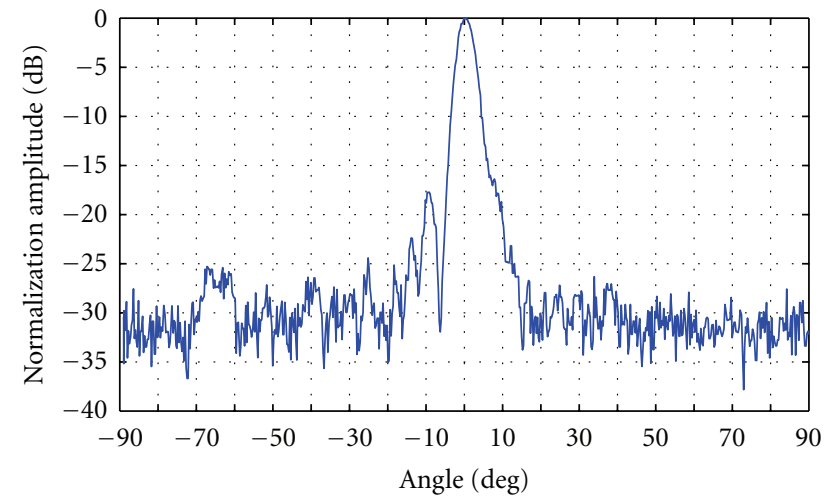

(a)

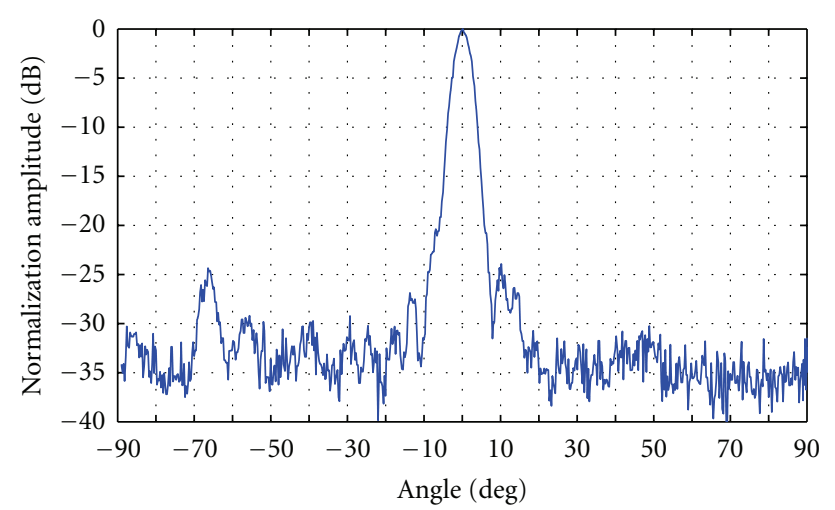

(b)

FIGURE 7: Antenna radiation pattern of the AbTR radar sample. (a) Vertical plane and (b) horizontal plane.

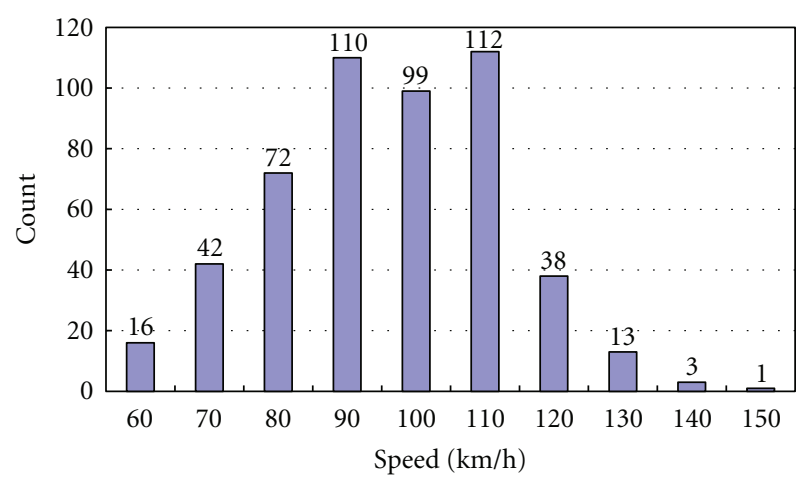

FIGURE 8: Speed distribution of the valid test results.

antenna beamwidths in the vertical and horizontal plane provided by the manufacturer are both less than $6^{\circ}$. Antenna radiation patterns of the sample in the vertical and horizontal planes are measured in the anechoic chamber at the Traffic Speed Lab of the National Institute of Metrology, China. The antenna radiation pattern in the vertical plane is shown in Figure 7(a); vertical beamwidth of the AbTR radar sample is $5.1^{\circ}\left( \pm 0.1^{\circ}\right)$, and the sidelobe level is $-17.7 \mathrm{~dB}( \pm 0.1 \mathrm{~dB})$ by calculation. The antenna radiation pattern in the horizontal plane is shown in Figure 7(b); horizontal beamwidth of the

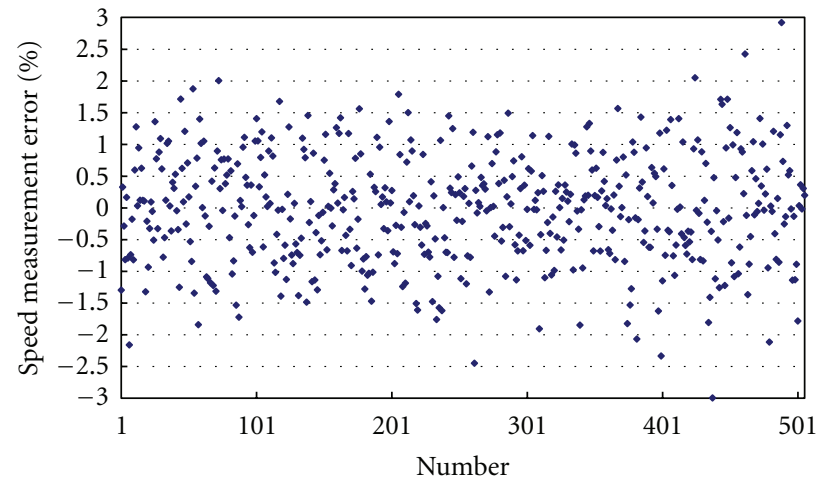

(a)

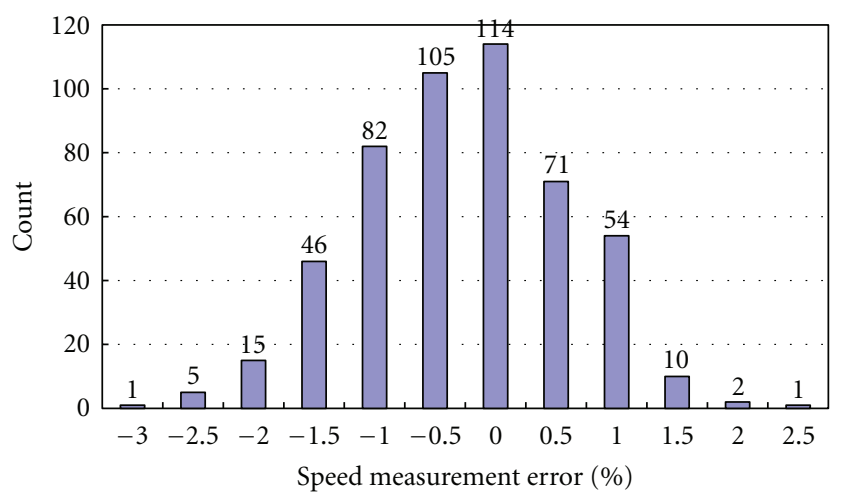

(b)

FIGURE 9: Relative measurement errors of the 506 valid target vehicles. (a) Distribution diagram and (b) statistics diagram.

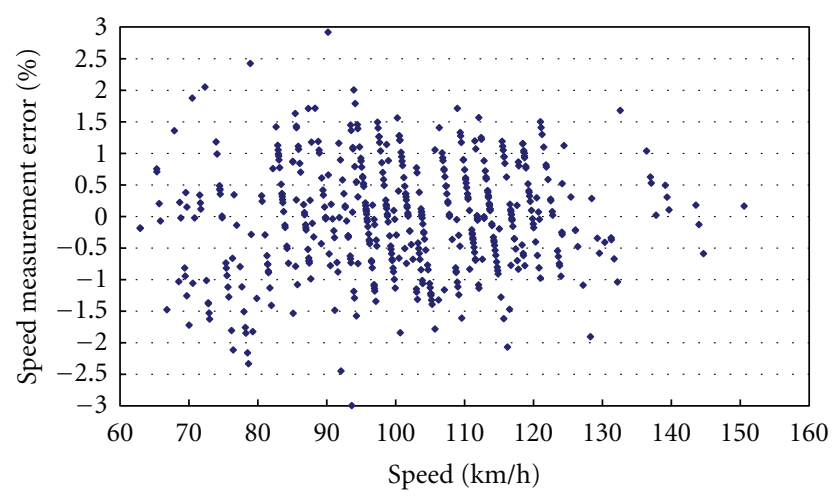

FIGURE 10: Relation between the relative measurement errors and target vehicles' speed.

AbTR radar sample is $5.2^{\circ}\left( \pm 0.1^{\circ}\right)$, and the sidelobe level is $-23.9 \mathrm{~dB}( \pm 0.1 \mathrm{~dB})$ by calculation.

There are a total of 517 target vehicles passing the metrological test region in this field test, in which the number of the valid test results is 506 . The effective rate of vehicle detection is about $98 \%$. Among the 11 invalid test results, 10 target vehicles travelled between the two lanes, and 1 target vehicle braked when passing the metrological test region. The AbTR radar sample did not detect any vehicles travelling in the adjacent two lanes. The speed distribution of the valid 


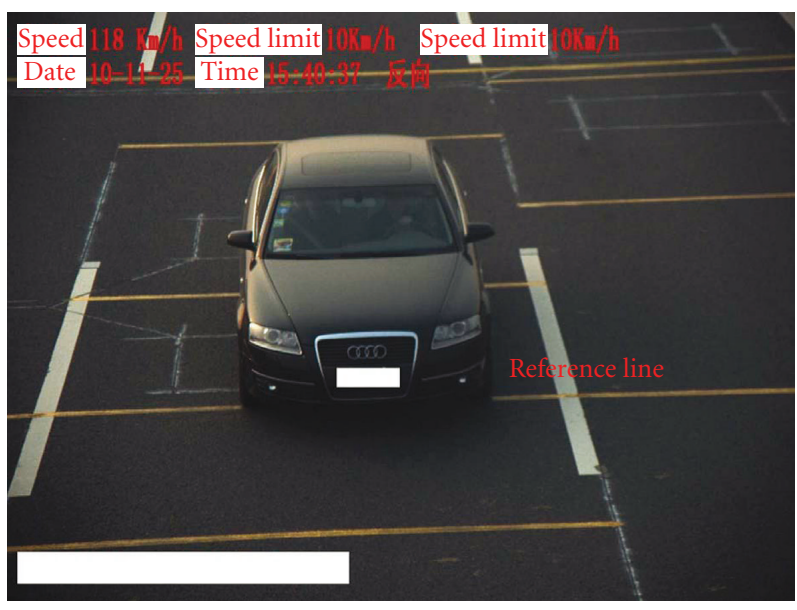

(a)

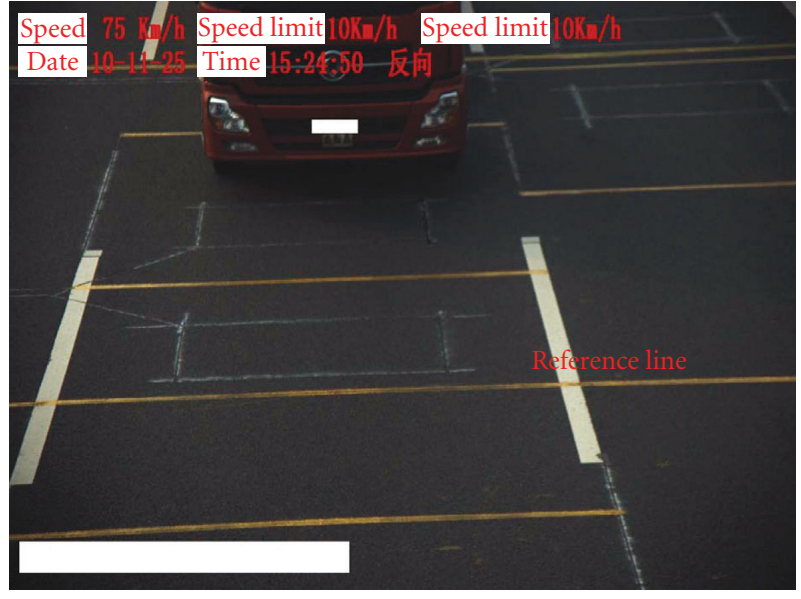

(b)

Figure 11: Photos for evidence of the selected 2 target vehicles. (a) A car; (b) a heavy truck.

test results is shown in Figure 8 , in which the minimum speed is nominally $62.9 \mathrm{~km} / \mathrm{h}$, and the maximum speed is nominally $150.6 \mathrm{~km} / \mathrm{h}$. Approximately $65 \%$ of the 506 valid target vehicles travel at the speed range from $90 \mathrm{~km} / \mathrm{h}$ to $120 \mathrm{~km} / \mathrm{h}$.

The distribution and statistics of the relative measurement errors of the 506 valid test results are shown in Figure 9. Figure 9 (a) shows that the relative measurement errors of the valid target vehicles are all distributed between $-3 \%$ and $3 \%$, in which the maximum positive error is approximately $2.9 \%$, and the minimum negative error is approximately $-3.0 \%$. The AbTR radar sample can meet the requirements for speed accuracy of field operation in China and is well qualified to the evidentiary purposes in traffic speed enforcement cases. Figure 9(b) gives the statistics diagram of the relative measurement errors in this field test and shows that the relative measurement errors of the 506 valid test results nominally exhibit a normal distribution.

The relation between the relative measurement errors and target vehicles' speed is shown in Figure 10. The relative measurement error of the target vehicle with low speed is larger than the target vehicle with high speed in general. By checking the evidence photograph, we find out that the target vehicle with low speed is generally a heavy truck and the target vehicle with high speed is generally a car. The reason has been analyzed in Section 4.1 that the error between the triggering angle $\varphi_{2}$ and the installation angle $\theta$ is larger than the one between the triggering angle $\varphi_{1}$ and the installation angle $\theta$ since the backscatter RCS of the heavy truck is larger than the car, which results in the larger relative measurement error according to (5). An example to validate the above theoretical analysis is given in Figure 11, where (a) shows the evidential photo of a car and (b) shows that of a heavy truck. The key information of target vehicles in the two photos is hidden to protect the privacy of drivers. Reference line in Figure 11 is corresponding to the ground position of the installation angle $\theta$, as shown in Figure 1. Obviously, the triggering line of the car is more closer to the reference line than that of the heavy truck, which means the triggering angle $\varphi_{1}$ of the car is closer to the installation angle $\theta$ than the triggering angle $\varphi_{2}$ of the heavy truck. The reference speed of the car is $118.1 \mathrm{~km} / \mathrm{h}$, and the measured speed by the AbTR radar is $118 \mathrm{~km} / \mathrm{h}$. The relative measurement error of the car is approximately $-0.1 \%$ according to (5). However, the reference speed of the heavy truck is $76.4 \mathrm{~km} / \mathrm{h}$, and the measured speed is $75 \mathrm{~km} / \mathrm{h}$. The relative measurement error of the heavy truck is approximately $-1.8 \%$, which is much larger than that of the car.

\section{Conclusions}

This paper has analyzed the reasons for the speed measurement error in AbTR radar field operation, studied the speed measurement error caused by antenna vertical beamwidth in theory, and calculated the relative error of speed measurement in various combinations of different vertical beamwidths and possible installation angles of AbTR radar in China. The conclusion of theoretical analysis is that the vertical beamwidth of certificated AbTR radar devices should not exceed $6^{\circ}$ to meet the requirement for speed measurement relative error of field operation within $\pm 3 \%$. The recommended requirements for the antenna horizontal beamwidth to operate with less interference from adjacent lanes have also been theoretically calculated with different installation heights and angles of AbTR radar in this paper. The conclusion is that the antenna horizontal beamwidth of the certificated AbTR radar devices should not exceed $6^{\circ}$ to meet the different installation requirements in China. A validation experiment in actual traffic has been performed on an AbTR radar sample with vertical beamwidth $5.1^{\circ}$ $\left( \pm 0.1^{\circ}\right)$ and horizontal beamwidth $5.2^{\circ}\left( \pm 0.1^{\circ}\right)$, respectively. The field test results have validated that the requirement of less than $6^{\circ}$ for AbTR radar antenna beamwidths in vertical and horizontal directions is a precondition for accurate and reliable field measurements. 


\section{Acknowledgments}

This work was supported in part by China Postdoctoral Science Foundation under Contract no. 20110490469, program funded by General Administration of Quality Supervision, Inspection and Quarantine of the People's Republic of China under Contract no. AHY0703 and program funded by National Institute of Metrology, P.R. China under Contract no. 22-JB1132. The authors would like to give special thanks to their colleagues in ROBOT Visual Systems GmbH, Germany for their support and cooperation in building up the field measurement standard system in China. They also would like to thank the academic editor and anonymous reviewers for their helpful comments which greatly improved the paper.

\section{References}

[1] International Association of Chiefs of Police, "Speedmeasuring device performance specifications: across-the-road radar module," Across-the-Road Radar Performance Standards, vol. 1, 2004.

[2] C. M. Weil, D. Camell, D. R. Novotny, and R. T. Johnk, "Across-the-road photo traffic radars: new calibration techniques," in Proceedings of the 15th International Conference on Microwaves, Radar and Wireless Communications (MIKON '04), vol. 3, pp. 889-892, May 2004.

[3] International Association of Chiefs of Police, "Speedmeasuring device performance specifications: down-the-road radar module," Down-the-Road Radar Performance Standards, vol. 1, 2004.

[4] J. Jendzurski and N. G. Paulter, "Calibration of speed enforcement down-the-road radars," Journal of Research of the National Institute of Standards and Technology, vol. 114, no. 3, pp. 137-148, 2009.

[5] OIML, "Radar equipment for the measurement of the speed of vehicles," OIML R 91, 1990.

[6] F. Eugene Knott, F. John Shaeffer, and T. Michael Tuley, Radar Cross Section, SciTech, Raleigh, NC, USA, 2004.

[7] L. Jian-hui, "The problems analysis in the verification of automatic monitor system for vehicles speeding," China Metrology, no. 7, pp. 112-113, 2009 (Chinese).

[8] L. Du, Q. Sun, C. Cai et al., "Introduction to the pattern approval standard equipment of vehicle speed-measuring devices in China and analysis of the field test data," China Metrology, no. 1, pp. 73-76, 2012 (Chinese).

[9] L. Du, Q. Sun, C. Cai et al., "Standard equipment for pattern approval field test of vehicle speed-measuring devices for traffic law enforcement in China," in Proceedings of the 8th International Symposium on Precision Engineering Measurements and Instrumentation (ISPEMI '12), Chengdu, China, August 2012.

[10] BIPM, IEC, ISO et al., "Evaluation of measurement dataguide to the expression of uncertainty in measurement," JCGM 100:2008, 2008. 

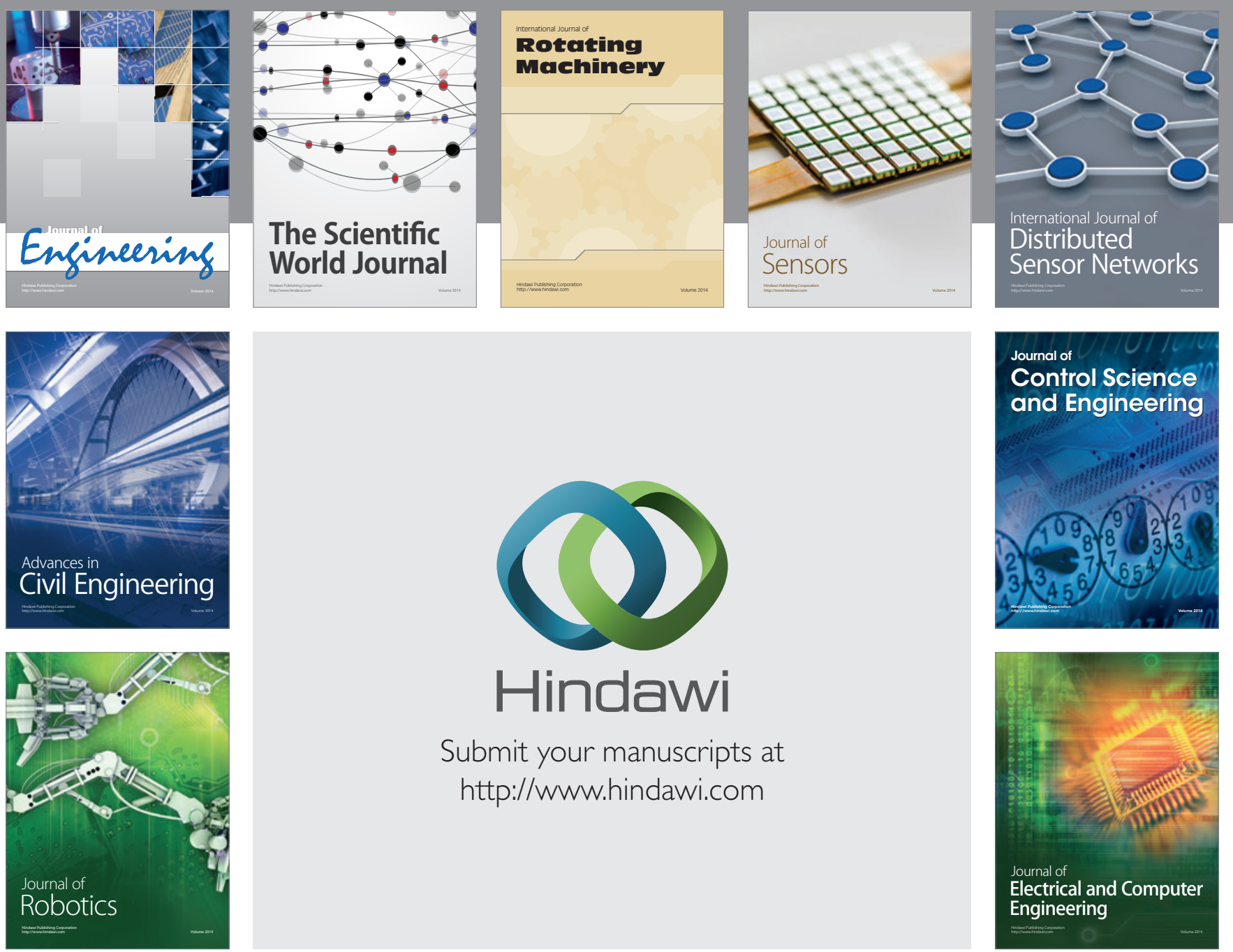

Submit your manuscripts at

http://www.hindawi.com
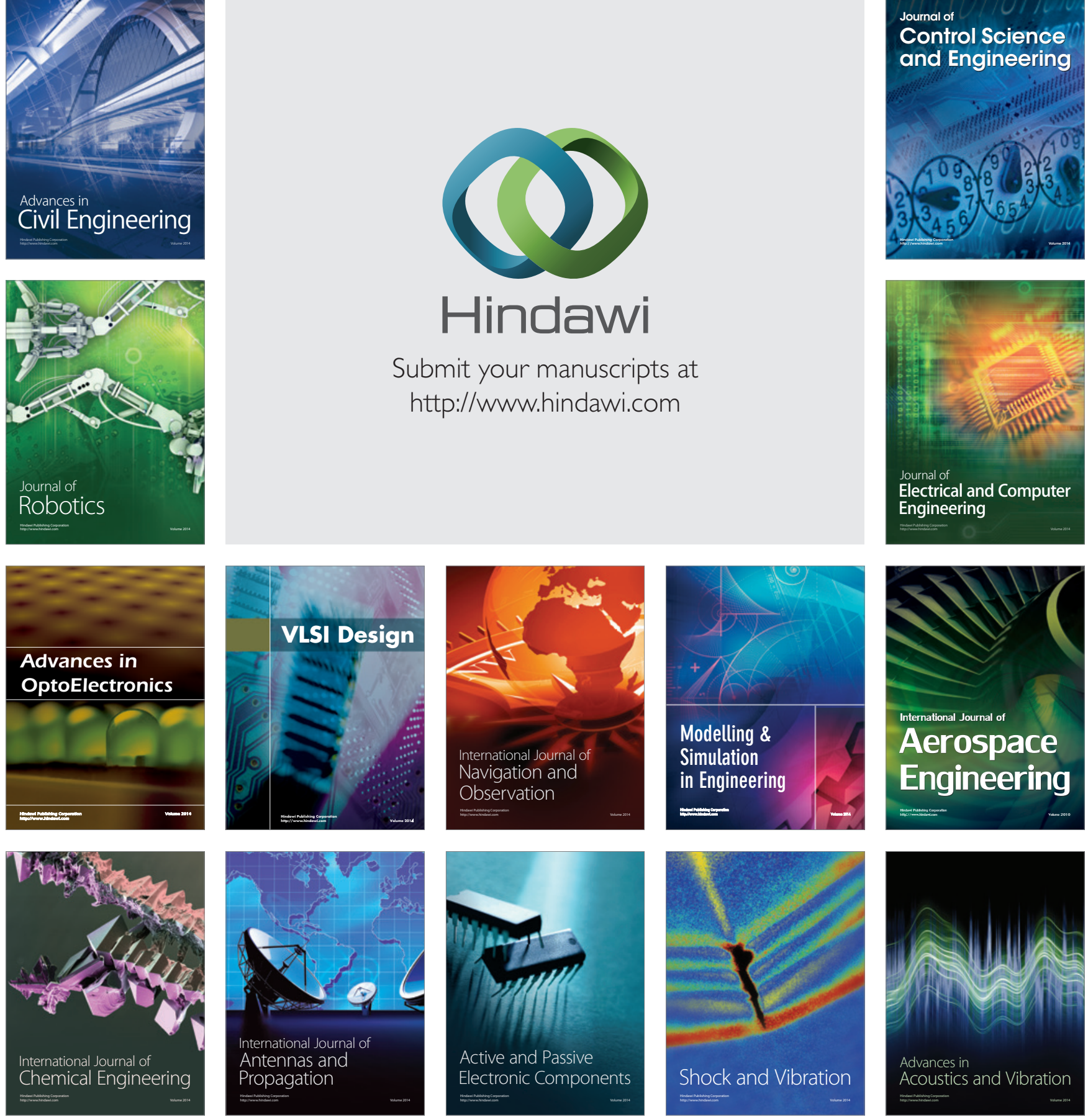